\title{
Análisis de Accesibilidad a Depósitos de Residuos Sólidos. El Caso de las Escombreras en la Ciudad de Manizales- Colombia
}

\author{
Diego A. Escobar ${ }^{(1)}$, Sebastián Martínez ${ }^{(1)}$ y Carlos A. Moncada ${ }^{(2)}$ \\ (1) Fac. de Ingeniería y Arquitectura, Depto. de Ing. Civil. Univ. Nacional de Colombia - Sede Manizales, \\ Colombia. Carrera 27 №64-60, Campus Palogrande, Edificio de Posgrados, Manizales, Colombia. (e- \\ mail: daescobarga@unal.edu.co; semartinezca@unal.edu.co) \\ (2) Fac. de Ingeniería, Depto. de Ing. Civil y Agrícola. Univ. Nacional de Colombia - Sede Bogotá, \\ Colombia. Carrera 30 N45-03, Campus Ciudad Universitaria, Edificio 214, Bogotá , Colombia. (e-mail: \\ camoncadaar@unal.edu.co)
}

Recibido Ago. 29, 2016; Aceptado Nov. 8, 2016; Versión final Dic. 27, 2016, Publicado Jun. 2017

\begin{abstract}
Resumen
En esta investigación se relaciona la ubicación de las escombreras en la ciudad de Manizales, con base en su accesibilidad, que está determinada por la red de infraestructura vial. Se cuenta con un total de siete puntos donde es posible almacenar escombros provenientes de obras civiles en construcción. Con la ayuda y utilización del software ArcGIS ${ }^{\circledR}$ y de las herramientas internas de Spatial Analyst ${ }^{\circledR}$ y Geostatistical Analyst ${ }^{\circledR}$ se aplica el método de los caminos mínimos (Kriging Ordinario); con los resultados obtenidos e información de la ciudad de Manizales, se plantean tiempos de cobertura de las variables área, población y vivienda en toda la zona urbana de la ciudad. Además, se identificaron geográficamente las obras civiles en ejecución para relacionar tiempos medios mínimos de viaje al depósito de escombros más cercano. Finalmente se hace una comparación entre los sitios de escombreras que se encuentran en funcionamiento y los nuevos designados por la ley, obteniendo un mejor panorama y comprensión de la situación actual en la ciudad de Manizales.
\end{abstract}

\section{Analysis of Accessibility to Solid Waste Disposal Sites. The case of the Debris Dumps in the City of Manizales-Colombia}

\begin{abstract}
In this research the location of debris dumps in the city of Manizales is analyzed, based on their accessibility, which is determined by the network of road infrastructure: The city has a total of seven legal points where it is possible to store debris from construction sites. The analysis was developed with the help and use of ArcGIS software, internal tools from Geostatistical Analyst $\Theta$ and Spatial Analyst $\Theta$. The method of minimum paths (Ordinary Kriging) including information from the city of Manizales such as population and housing throughout the urban area to calculate accessibility by time coverage of the city area. Also, construction sites and projects where geographically located to relate average minimum travel time to the nearest debris' dump. Finally a comparison between dump sites that are in operation and new ones permitted by law is done, to obtain a better view and understanding of the current situation in the city of Manizales.
\end{abstract}




\section{INTRODUCCIÓN}

Los municipios de Manizales y Villamaría se ubican geográficamente sobre la cordillera central en la región centro occidente de Colombia (ver Fig. 1) y para la zona de estudio (Ver Fig. 2); ambos municipios conforman una conurbación que alberga aproximadamente 416.000 habitantes en un área que se caracteriza por su topografía quebrada y abrupta.

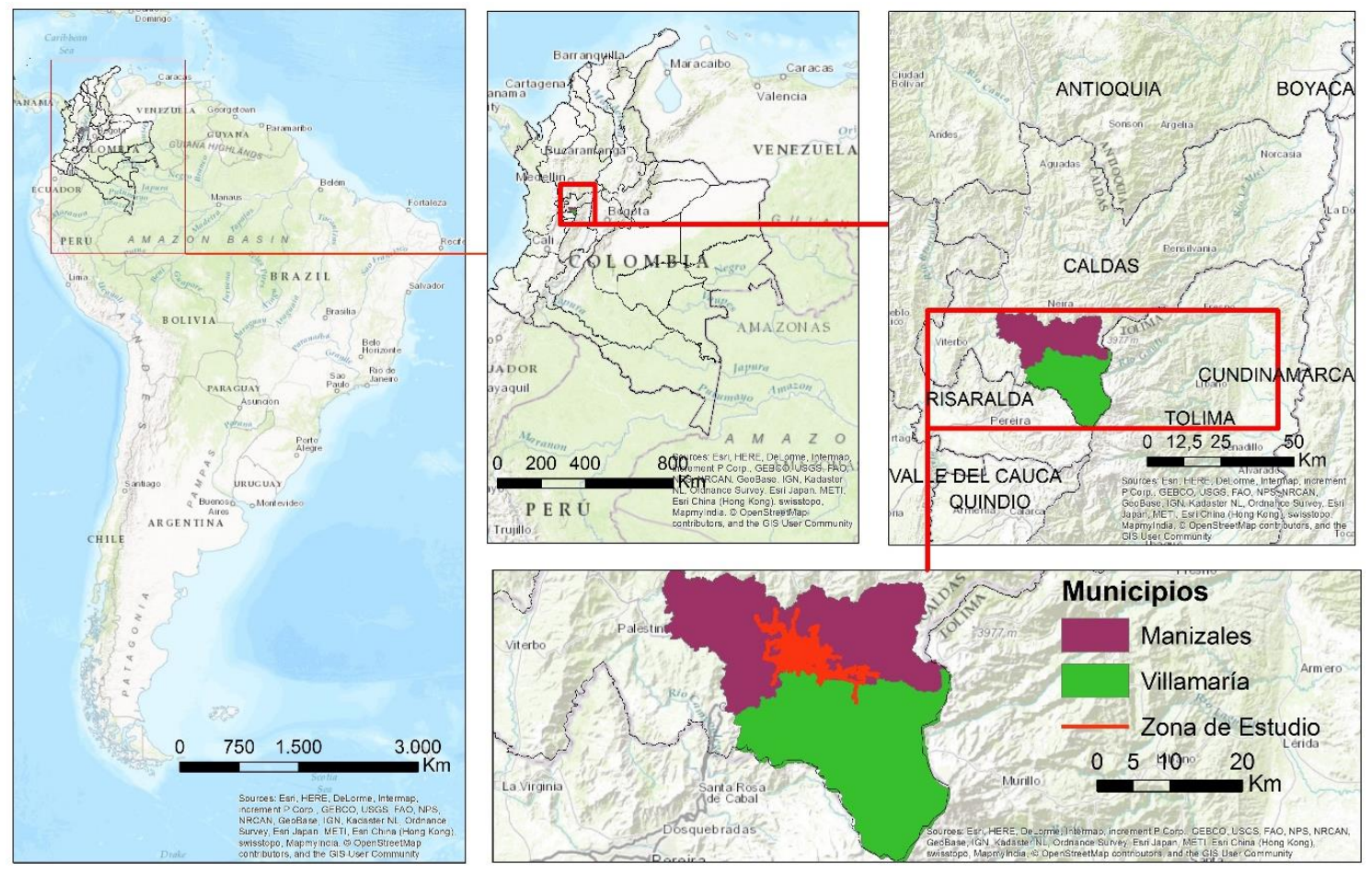

Fig. 1: Ubicación de Manizales y Villamaría partiendo de Suramérica.

De acuerdo al Plan de Ordenamiento Territorial vigente para la ciudad de Manizales (POT, 2007), la ciudad cuenta con seis (6) sitios legalmente autorizados para almacenar escombros o residuos desechables de construcción de obras civiles; de igual forma, el municipio de Villamaría cuenta con una (1) escombrera habilitada por el Plan de Gestión Integral de Residuos Sólidos del municipio (PGIRS, 2015). No obstante, mediante el Decreto №389 de 2015, se legalizó la adecuación y puesta a punto dos escombreras, una ubicada en el Barrio la enea (Finca la Granja), y otra en el Barrio La Playita (Ver Fig. 2).

Los análisis de accesibilidad se requieren para detallar la infraestructura vial y la movilización de cada uno de los vehículos en la ciudad, en búsqueda de la mejor combinación entre usos del suelo, estrategias de transporte y desarrollo urbano (Geurs Van Wee, 2004). Al transcurrir la historia del planeamiento regional y urbano, la concepción de "accesibilidad" ha sido transcendentalmente importante, ya que se conocen aplicaciones desde la mitad del siglo pasado, periodo que se usó para estudios relacionados con planeación económico-regional y teorías de localización (Batty, 2009). El término accesibilidad establece la facilidad de comunicación que puede existir o existe entre determinados sectores, comunidades o actividades, la cual es posible dada la oferta de variados modos de transporte (Morris et al. 1978). Es importante conocer y comprender la accesibilidad como una necesidad primaria (Halden, 2011), ya que por medio de ésta es se pueden explicar las posibilidades existentes para llegar a diversas actividades (empleo, salud, educación, escombreras, etc.). Este concepto también se relaciona con ciencias como: el turismo (Kastenholz et al., 2012), el desarrollo económico (Mackinnion et al., 2008), la operatividad de modos de transporte (Escobar y García, 2012), el análisis demográfico (Kotavaara, 2011), el análisis de cohesión social (López et al., 2008), la sostenibilidad (Vega, 2011) y para las algunas áreas del conocimiento tan nuevas como las redes sociales (Sailer et al., 2012).

La actividades industriales como la construcción de infraestructura, son esenciales para el desarrollo y evolución de las ciudades; sin embargo, es una actividad que genera un importante impacto sobre el medio ambiente, teniendo como uno de los aspectos más preocupantes el de la generación de residuos dados los procesos de construcción, remodelación y demolición de infraestructura (Castaño et al., 2013), situación que ha venido generando una mayor conciencia social ambiental, incentivando la realización de gestiones ambientales y planes sostenibles para la adecuada disposición de dichos residuos (Achillas et al., 2013); Así mismo, se ha identificado que es en el entorno urbano donde se producen los principales volúmenes de 
residuos, dado el crecimiento progresivo y constante de las ciudades, el mejoramiento de la calidad de vida de la población, los nuevos avances tecnológicos en materia de infraestructura y el desarrollo de las nuevas construcciones (Leandro, 2008). Generalmente se encuentra que el escombro se compone principalmente por $50 \%$ material de albañilería (cerámica, escayolas, escorias, entre otros), $20 \%$ hormigón, un $10 \%$ de asfalto y un $20 \%$ de otros materiales como maderas, hierros y vidrio (Serrano y Pérez, 2009).

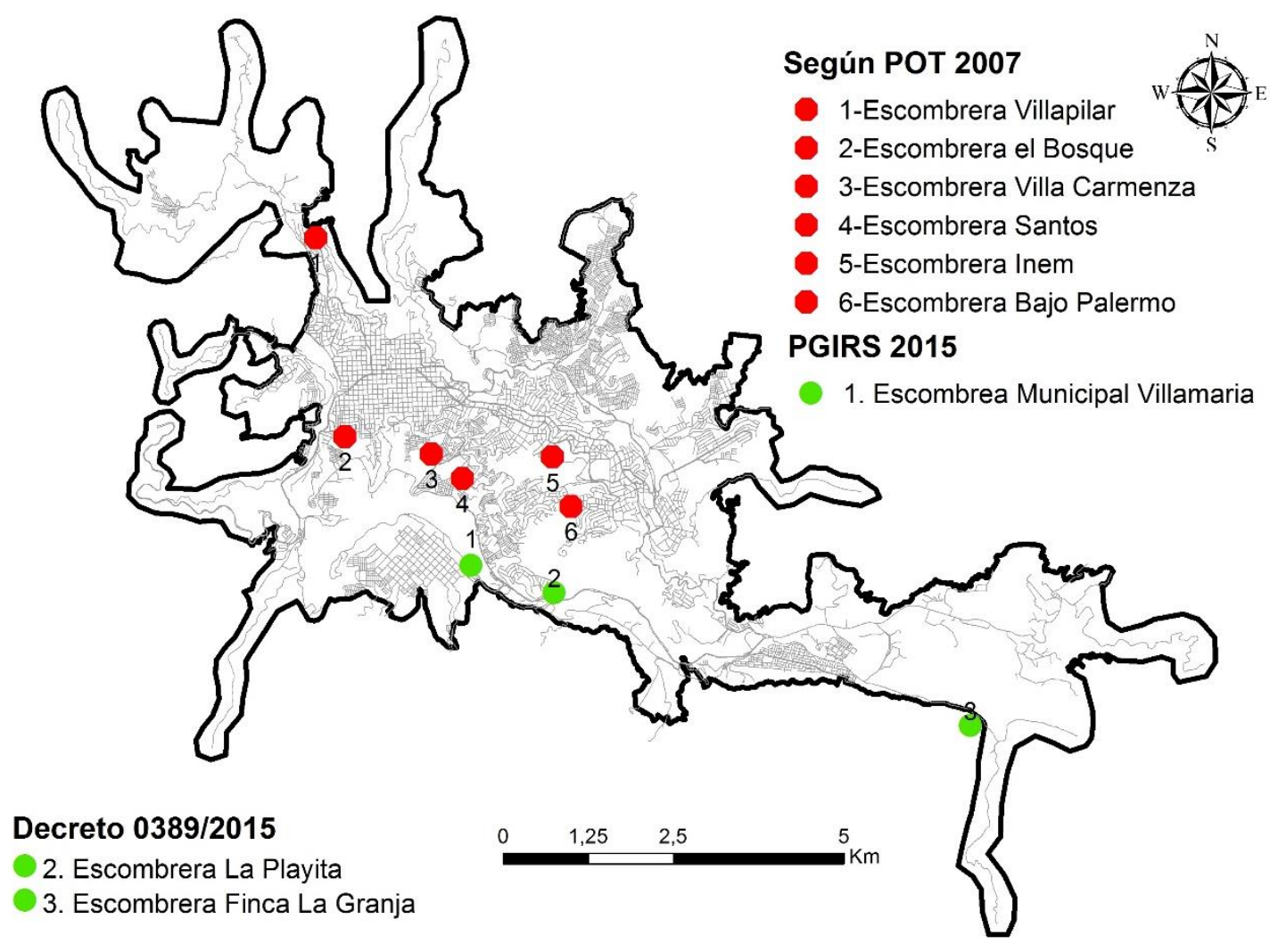

Fig. 2: Escombreras de Manizales y Villamaría; Operando actualmente las N² y №3 por Decreto 0389/2015.

En relación con los estudios de ubicación de sitios para la disposición de residuos sólidos y escombros, se ha encontrado que éstos se basan principalmente en análisis ambientales que detallan la composición química de los lixiviados provenientes de dichos residuos (Hernández et al., 2013) y su impacto en los sectores donde se pretende aprobar la prestación de dicho servicio. Una ciudad en Colombia con características demográficas y geoespaciales similares al caso de estudio que aquí se abordó, es la ciudad de Neiva (Huila), en la cual se tienen aprobados siete puntos de recepción de escombros, que actualmente funcionan en la ciudad (Sánchez, 2013); así mismo, la ciudad de Pitalito (Huila), dispone actualmente de un sitio destinado para el almacenamiento de escombros, no obstante, existe un estudio que define tres posibles sitios de disposición de este tipo de material (Empitalito E.S.P.,2014). En ambos casos, las variables de definición de estos sitios obedecen más a características de tipo ambiental, topográfico y de capacidad de almacenamiento, que a características relacionadas con la red de transportes que permiten llevar dichos escombros a un sitio o sitios determinados.

De acuerdo con la literatura internacional; se destaca que en los Países Bajos, el reciclaje de residuos de construcción se enfoca en la logística y en la localización adecuada para optimizar procesos de reciclaje (Barros et al., 1998); en Lisboa, Portugal, se separan los residuos de construcción y demolición y se estudian las implicaciones económicas del reciclaje de dichos residuos, proponiendo plantas de reciclaje a gran escala para mitigar los impactos ambientales (Coelho y De Brito, 2013); en la región de Macedonia Central, Grecia, se proponen metodologías de análisis multicriterio, que proporcionan información de la ubicación óptima (económica, ambiental y social) de unidades de gestión alternativas de residuos de construcción y demolición (Banias et al., 2010); por su parte, la industria canadiense, propone estrategias sostenibles enfocadas en $3 R$ (reducir, reutilizar y reciclar) y así minimizar la eliminación de los residuos de construcción, aplicándose un índice de sostenibilidad C \& D basado en el ciclo de vida (Yeheyis et al., 2013). Las mega ciudades del futuro, por ejemplo Ho Chi Minh City, Vietnam, se encuentra en la tarea de facilitar la implementación de tecnologías adicionales para el tratamiento de este tipo de residuos, buscando la forma de volverlos rentables y sostenibles (ThiKimOanh et al., 2015). 
Se ha encontrado también que en los países en desarrollo, se apoyan de los Sistemas de Información Geográfica (SIG) para estudiar aspectos de selección, optimización de rutas y aceptación pública para encontrar el sitio adecuado de disposición final de residuos, que genere el mínimo impacto social (Khan y Samadder, 2014); vale la pena destacar la República de Serbia con estudios enfocados al campo práctico de la recolección de los residuos generados en construcción, basándose en un proceso de jerarquía analítica (AHP) y enfatizando que el impulso institucional-administrativo es el más importante para el desarrollo de un sistema de gestión de residuos sostenible (Tot et al., 2016).

No se encuentra en la literatura relacionada con el tema, investigaciones que aborden directamente el análisis de este tipo de servicios o equipamientos desde el punto de vista de accesibilidad territorial urbana y su interacción con la red de infraestructuras del transporte. Por tal razón, se presenta este estudio utilizando la red vial, la cual es un insumo necesario para el cálculo de accesibilidad, la cual metodológicamente apoya el análisis espacial de equipamientos en relación con cualquier nodo especifico de una red (Curl et al., 2012).

Para Colombia, la accesibilidad, analizada desde un punto de vista geográfico, ha sido poco estudiada, desaprovechando el innumerable potencial que posee este tipo de metodologías; a pesar de tal deficiencia, se poseen ejemplos reales de aplicación de análisis de accesibilidad a escala regional (Escobar et al., 2013) y a escala urbana (Younes et al., 2016; Escobar et al., 2015, 2012; Botero et al., 2011). A continuación se abordarán los capítulos de metodología aplicada, formulación del modelo geoestadístico y finalmente se discuten los resultados y se presentan las principales conclusiones.

\section{METODOLOGÍA}

La metodología de la investigación se resume en tres etapas principales (Ver Fig. 3), a saber: 1) Actualización y validación de características operativas de la red de infraestructura del transporte para los cálculos de Accesibilidad Media Integral, geoespacialización de escombreras y ubicación de nuevas construcciones; 2) Cálculo de la Accesibilidad Media Integral, desde todos los nodos de la red a las escombreras; y 3) Análisis de cobertura de área, población, vivienda y construcción de obras civiles en relación con la ubicación de la escombreras.

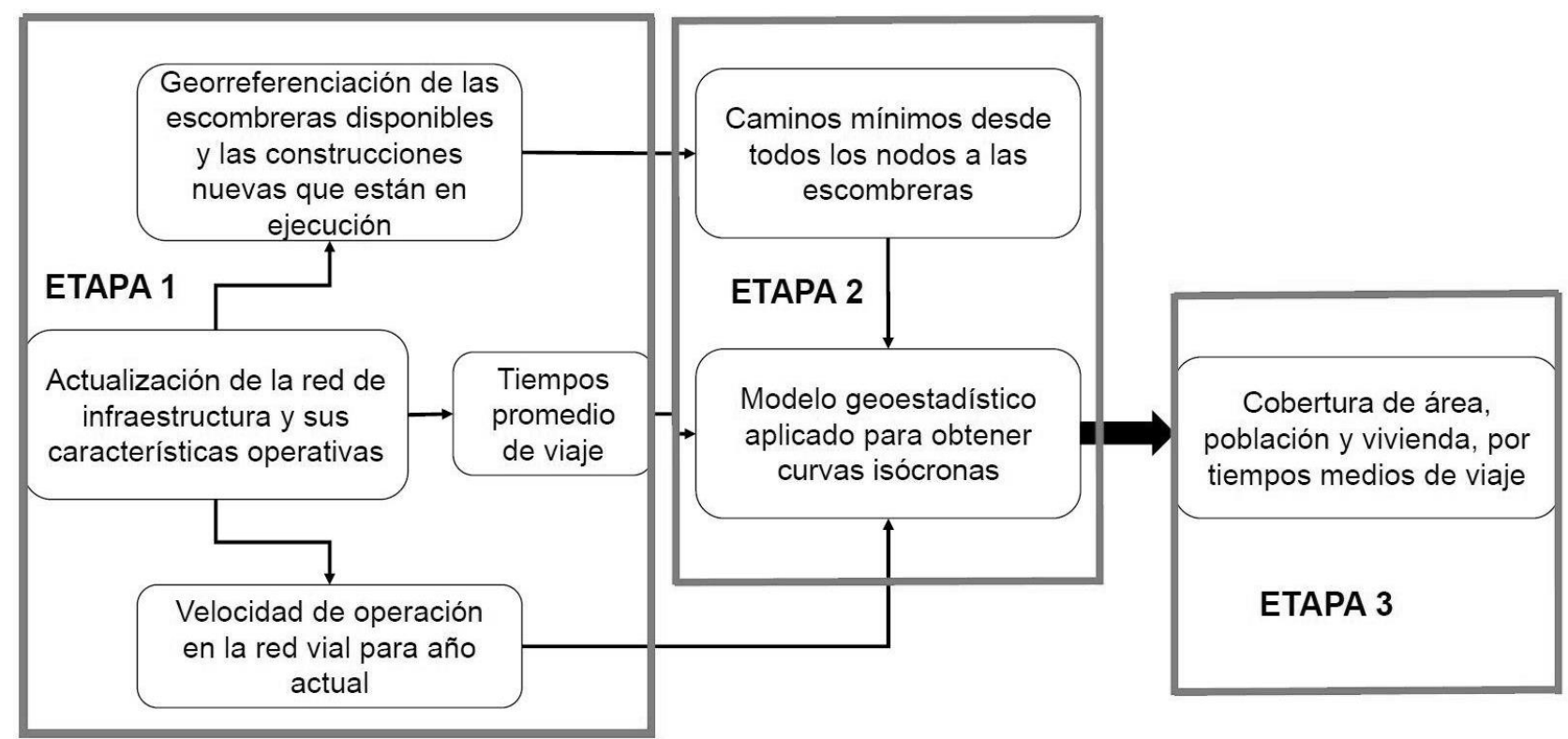

Fig. 3: Metodología aplicada.

Etapa 1. Actualización y validación de características operativas de la red de infraestructuras del transporte para los cálculos de Accesibilidad Media Integral, la geoespacialización de las escombreras y la ubicación de obras nuevas: En esta etapa se tomó como información primaria la red de infraestructura del transporte de la ciudad de Manizales (pública y privada), incluyendo las características operativas de la misma (velocidades de operación), mediante el método de vehículo flotante y dispositivos GPS instalados en diferentes modos de transporte (auto, taxi, motocicleta, camión y transporte público urbano). Los datos de GPS se almacenaron y digitalizaron con su respectiva localización (longitud, latitud) para obtener la velocidad promedio existente entre cada par de nodos (intersección de segmentos) unidos por un arco (segmento de red). Posteriormente, con la información oficial de los Planes de Ordenamiento Territorial para 
la ciudad de Manizales y el municipio de Villamaría, se geoespacialización los puntos de escombreras en los cuales se entiende que son los lugares que actualmente funcionan para el depósito de residuos provenientes de las construcciones. Por otro lado, se tomó información de la web ConstruOferta.com, donde se ubican todas las construcciones de obra civil que se encuentran en ejecución en el área de estudio; se toma como supuesto que serán dichas construcciones nuevas las que mayor demandan el uso de las escombreras municipales.

Etapa 2. Cálculo de la Accesibilidad Media Integral, desde todos los nodos de la red a los puntos de escombreras: En esta etapa, se aplican modelos geoestadísticos aprovechando la capacidad de los Sistemas de Información Geográfica (SIG), los cuales se relacionan directamente con el uso de algoritmos (Zhang et al., 2009) como el de caminos mínimos. Se realiza el cálculo de Accesibilidad Media Integral, mediante los caminos mínimos entre todos los nodos de la red hasta los puntos de las escombreras. La accesibilidad se estudia a partir de un vector de tiempo medio de viaje ( $\left.T v_{i}\right)$ que, de acuerdo a las características de la red, permite generar las curvas isócronas. Con la ayuda del software ArcGIS $\AA$ y de las herramientas internas de Network Analyst® y Geostatistical Analyst $\AA$ del aplicativo ArcMap® de ESRI fue aplicado el modelo.

Etapa 3. Análisis de cobertura de área, población, vivienda y construcción de obras civiles en relación con la ubicación de las escombreras: Se trata de la etapa final, donde se analizan los tiempos medios de viaje para cada uno de los sectores en cuestión, con el cálculo de los porcentajes de área, población y vivienda; por otra parte, analizar las construcciones que se están ejecutando en la ciudad de Manizales, para así encontrar el recorrido mínimo a la escombrera más cercana. Por consiguiente, se realiza el análisis con las escombreras que se encuentran actualmente en funcionamiento y las de nueva apertura.

\section{FORMULACIÓN DEL MODELO}

El modelo que a continuación se formula, se basa en lo propuesto por Shen (2002); la Accesibilidad Media Integral se analizó a partir del vector tiempo medio de viaje ( $\left.T v_{i}\right)$, el cual representa el tiempo promedio de viaje desde el nodo $i$ hasta los puntos designados como las escombreras. En el cálculo se utilizó un algoritmo del SIG que permite calcular la menor impedancia (Caminos Mínimos) entre un nodo específico y los demás nodos de la red, conformando una matriz unimodal de impedancias. A través de esta matriz y conociendo la velocidad de operación promedio de cada arco, se elaboró la matriz de tiempos promedios mínimos de viaje, en la cual se minimiza el tiempo medio de viaje entre todos los nodos y cada punto específico designado como las escombreras, que están conformados en la totalidad de la red en estudio. Una vez determinada la matriz de tiempos promedios mínimos de viaje, se obtuvo el vector de tiempo promedio de viaje $T v_{i}\left(\right.$ Ver expresión (1)). Donde $T v_{i}=$ tiempo de viaje mínimo promedio entre el nodo $i$ y los demás nodos de la red $n=$ número de nodos de la red.

$\overline{T_{v l}}=\frac{\sum_{j=1}^{m} t_{v i}}{(n-1)}$, siendo $i=1,2,3, \ldots, n ; j=1,2,3, \ldots, m$.

El vector de Tiempos Mínimos de Viaje está representado por una matriz de tamaño [nx1], la cual se relaciona con las coordenadas (longitud y latitud) de cada nodo de la red, generándose una nueva matriz de tamaño [nx3]. El modelo geoestadístico Kriging ordinario con semivariograma lineal fue el escogido para la predicción de la variable TMV (Tiempo Mínimo de Viaje) a lo largo y ancho de la ciudad. Kriging Ordinario es un método geoestadístico utilizado para la estimación de valores desconocidos acerca de valores conocidos; con la creación de un semivariograma a partir de los puntos conocidos y en relación con el parámetro de ponderación de semivarianza media y así poder calcular los puntos desconocidos; la ecuación fundamental y básica del método se observa en la expresión (2); donde $Z_{0}$ es el valor a estimar, $Z_{x}$ son los valores de los puntos conocidos, $W_{x}$ es la correlación o pesos de los puntos conocidos respecto a los puntos y $\mathrm{S}$ son los números de puntos conocidos.

$Z_{0}=\sum_{i=1}^{S} Z_{x} w_{x}$

Para la semivarianza media se tiene que $h$ es la distancia entre puntos, $n$ son números pares de puntos que se definen según la distancia y z son los valores de puntos conocidos. Ver expresión (3). (Phatarapon V., et al, 2015).

$f(h)=\frac{1}{2 n} \sum_{i=1}^{n}\left[z\left(x_{i}\right)-z\left(x_{i}+h\right)\right]^{2}$

La metodología aplicada toma las velocidades de operación promedio de los arcos que componen la red, lo cual se establece como un efecto simplificador en relación con la aplicación del modelo propuesto, ya que 
se está homogenizando el tiempo medio de viaje de todos los modos de transporte en un solo valor; esta situación debe tenerse en cuenta al momento de tomarse los resultados del presente modelo como base en procesos de planificación.

\section{RESULTADOS Y DISCUSIÓN}

Para mayor claridad, los resultados son presentados y analizados en dos subsecciones: (i) Accesibilidad Media Integral a las Escombreras reglamentadas en POT 2007 y (ii) Accesibilidad Media Integral a las Escombreras. Situación real (2016).

\section{Accesibilidad Media Integral a las Escombreras reglamentadas en POT 2007}

Manizales cuenta con seis (6) escombreras ubicadas dentro del perímetro urbano y Villamaría con una (1), cada una de éstas se geoespacializó teniendo como fuente de información los Planes de Ordenamiento Territorial de ambos municipios, documentos considerados técnica y legalmente válidos. En la Fig. 4 se observan las curvas isócronas de Accesibilidad Media Integral obtenidas al analizar la ubicación de las escombreras legalmente establecidas; se observan los sectores que se encontrarían cubiertos por tiempos medios de viaje de entre 5 y 40 minutos. Se observa una cobertura más efectiva de los sectores centro y occidente, en relación con los otros sectores de la ciudad. En lafigura 4 se observa las construcciones de obras civiles que se encuentran actualmente en inicio de obra o que se presumen empezarán su construcción en un lapso de meses; se detalla que las construcciones nuevas se encuentran en un intervalo de 5 a 20 minutos, de acuerdo a las curvas isócronas de la accesibilidad media integral; se tiene en cuenta que se geoespacializaron un total de 40 obras nuevas, por consiguiente se analizó que un $90 \%$ de las construcciones nuevas se concentran entre el intervalo de 5 a 20 minutos; se tiene como tiempo mínimo de 20 minutos para hacer uso de cualquier sitio designado como escombrera.

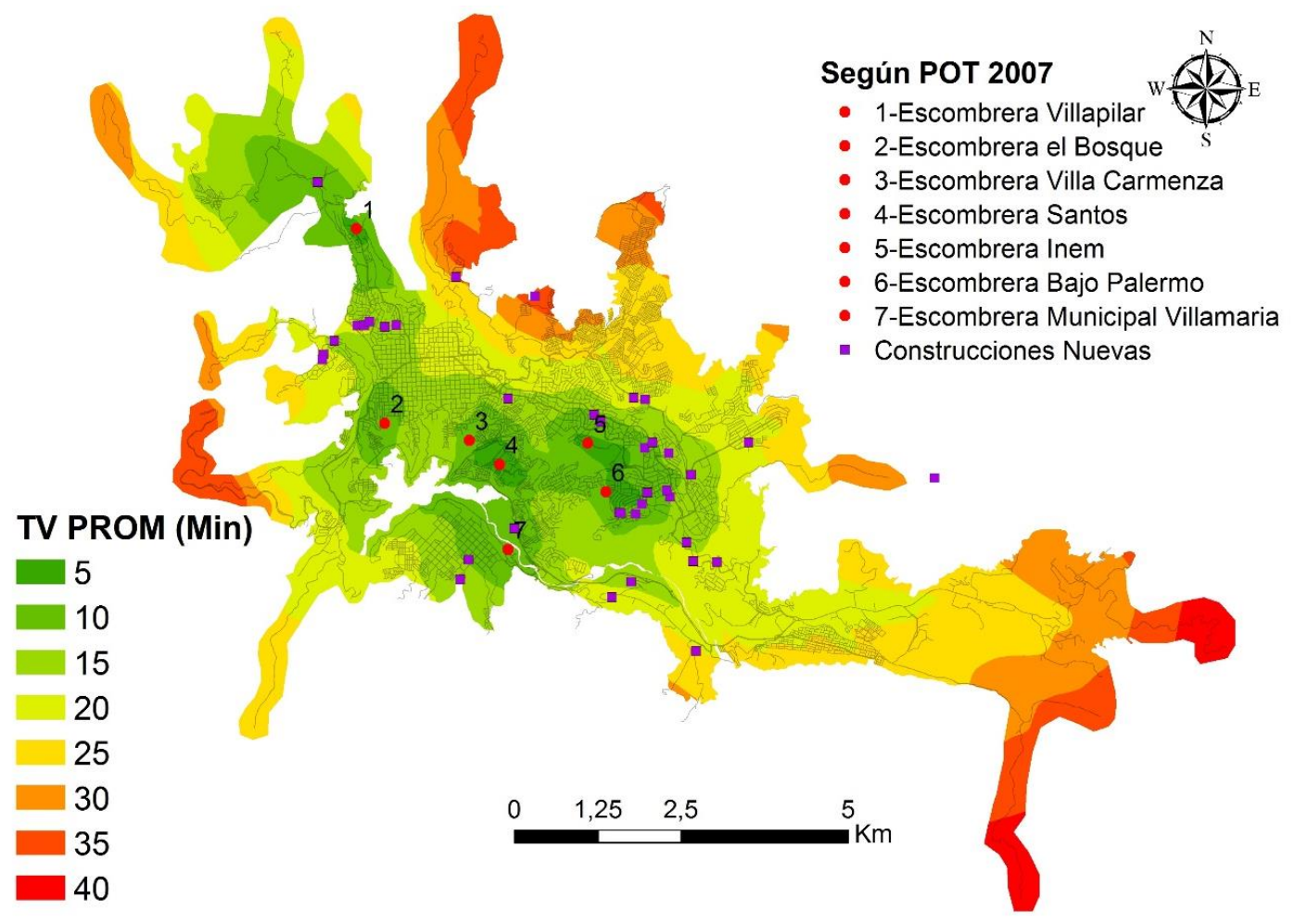

Fig. 4: Accesibilidad Media Integral y ubicación de las siete (7) escombreras oficiales en la zona de estudio.

En la Tabla 1 se presentan los valores obtenidos al relacionar las curvas isócronas de Accesibilidad media integral con datos sociodemográficos como población, número de viviendas y área. Se observa por ejemplo, que a partir de las escombreras oficiales, más del $55 \%$ de la población y del número de viviendas se cubrirían con tiempos medios de viaje de hasta 15 minutos, valor que disminuye a un $35 \%$ para el caso de la variable área, en el mismo período de tiempo.

En la Fig. 5 se presenta la distribución y variación de porcentajes de cobertura de las tres variables analizadas (área, población y número de viviendas), según las curvas isócronas de tiempo en intervalos de cinco minutos. Se observa que los mayores porcentajes de cobertura se obtienen para tiempos medios de 
viaje de entre 5 a 25 minutos para las tres variables. Por otra parte, se observa que para las variables población y número de viviendas hay mayores porcentajes de cobertura para menores tiempos medios de viaje, situación que no se observa en los porcentajes de cobertura relacionados con la variable área, lo cual se relaciona directamente con las características de densidad poblacional del área de estudio.

Tabla 1: Porcentaje de área, población y vivienda, cubiertas por las curvas isócronas de tiempo para la zona de estudio.

\begin{tabular}{|l|l|l|l|l|l|l|l|l|l|}
\hline \multirow{2}{*}{$\begin{array}{c}\text { Curva } \\
\text { Isócrona } \\
(\text { Min) }\end{array}$} & \multicolumn{4}{|c|}{ Área } & \multicolumn{4}{c|}{ Población } & \multicolumn{3}{c|}{ Vivienda } \\
\cline { 2 - 10 } & $\begin{array}{c}\text { Porcentaje } \\
(\%)\end{array}$ & $\begin{array}{c}\text { Acumulado } \\
(\%)\end{array}$ & Hab. & $\begin{array}{c}\text { Porcentaje } \\
(\%)\end{array}$ & $\begin{array}{c}\text { Acumulado } \\
(\%)\end{array}$ & $N^{\circ}$ Viv. & $\begin{array}{c}\text { Porcentaje } \\
(\%)\end{array}$ & $\begin{array}{l}\text { Acumu- } \\
\text { lado }(\%)\end{array}$ \\
\hline $0-5$ & 93,7 & $1,6 \%$ & $1,6 \%$ & 9.294 & $2,2 \%$ & $2,2 \%$ & 3.219 & $3,3 \%$ & $3,3 \%$ \\
\hline $5-10$ & 745,5 & $12,7 \%$ & $14,3 \%$ & 87.560 & $21,1 \%$ & $23,3 \%$ & 22.337 & $22,9 \%$ & $26,2 \%$ \\
\hline $10-15$ & $1.181,4$ & $20,1 \%$ & $34,4 \%$ & 139.749 & $33,7 \%$ & $57,0 \%$ & 33.052 & $33,8 \%$ & $60,0 \%$ \\
\hline $15-20$ & $1.359,8$ & $23,2 \%$ & $57,6 \%$ & 90.857 & $21,9 \%$ & $78,9 \%$ & 20.434 & $20,9 \%$ & $80,9 \%$ \\
\hline $20-25$ & $1.195,2$ & $20,4 \%$ & $78,0 \%$ & 74.863 & $18,0 \%$ & $96,9 \%$ & 15.299 & $15,7 \%$ & $96,6 \%$ \\
\hline $25-30$ & 724,1 & $12,3 \%$ & $90,3 \%$ & 11.860 & $2,9 \%$ & $99,8 \%$ & 3.043 & $3,1 \%$ & $99,7 \%$ \\
\hline $30-35$ & 439,4 & $7,5 \%$ & $97,8 \%$ & 940 & $0,2 \%$ & $100,0 \%$ & 281 & $0,3 \%$ & $100,0 \%$ \\
\hline $35-40$ & 129,0 & $2,2 \%$ & $100,0 \%$ & 0 & $0,0 \%$ & $100,0 \%$ & 0 & $0,0 \%$ & $100,0 \%$ \\
\hline & $5.868,1$ & $100,0 \%$ & & 415.124 & $100,0 \%$ & & 97.666 & $100,0 \%$ & \\
\hline
\end{tabular}

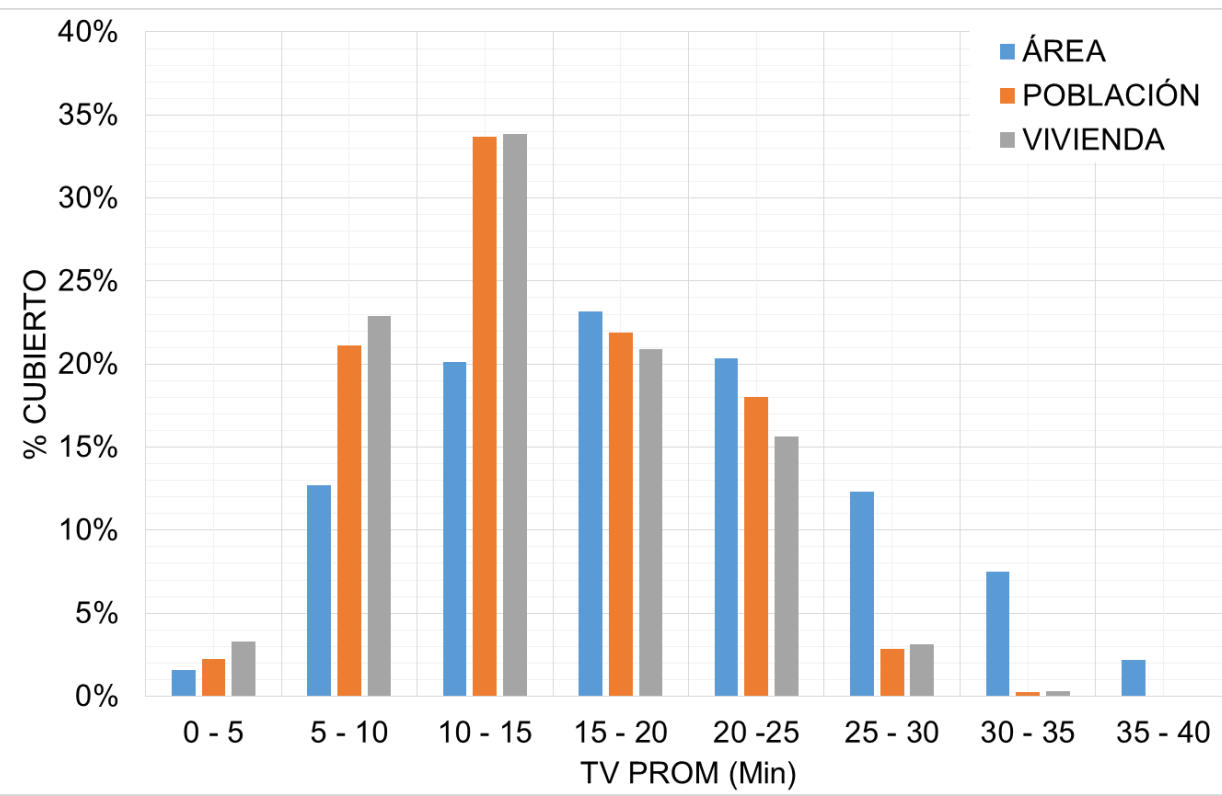

Fig. 5: Distribución porcentual de área, población y vivienda, cubiertas por las curvas isócronas.

Se aprecia en la Fig. 6 la ojiva porcentual acumulada de las variables área, población y vivienda que son cubiertas por las curvas isócronas. Es posible concluir que el $25 \%$ del área se cubre con tiempos medios de viaje de entre 10 y 15 minutos, mientras que el $25 \%$ de la población y vivienda se cubre con tiempos medios de viaje menores a los 10 minutos; así mismo, el 50\% del área se cubre a los 20 minutos y el 50\% de la población y la vivienda se cubren a los 15 minutos. Si se analizan los valores de estos dos percentiles (25\% y $50 \%$ ), se observa una pendiente más pronunciada entre los periodos de tiempo para las variables población y viviendas, lo que indica unas mejores condiciones de accesibilidad en sectores de alta densidad si se compara con la cobertura obtenida para la variable área.

Por otra parte, al relacionar las curvas isócronas obtenidas con el número de obras de construcción civil que se están ejecutando, se observa que para los intervalos presentados de 5 a 20 minutos donde están presenta el $90 \%$ de las nuevas construcciones, se cubren hasta el $75 \%$ de la población y la vivienda y un $65 \%$ del área estudiada.

Los casos anteriores, se basan en información oficial publicada en instrumentos de planificación territorial, no obstante, algunas de las escombreras legalmente establecidas y utilizadas para los anteriores cálculos, se encuentran actualmente clausuradas por haber alcanzado su capacidad de acumulación de material o 
por presentar importantes deficiencias funcionales en algunos componentes como las obras de drenaje. De las seis (6) escombreras que se encuentran reglamentadas en el POT del 2007 (Acuerdo 663 de 2007), actualmente ninguna de ellas funciona por variados motivos expuestos en las Resoluciones de Licencia Ambiental expedidas por la autoridad ambiental competente, CORPOCALDAS (Ver Tabla 2).

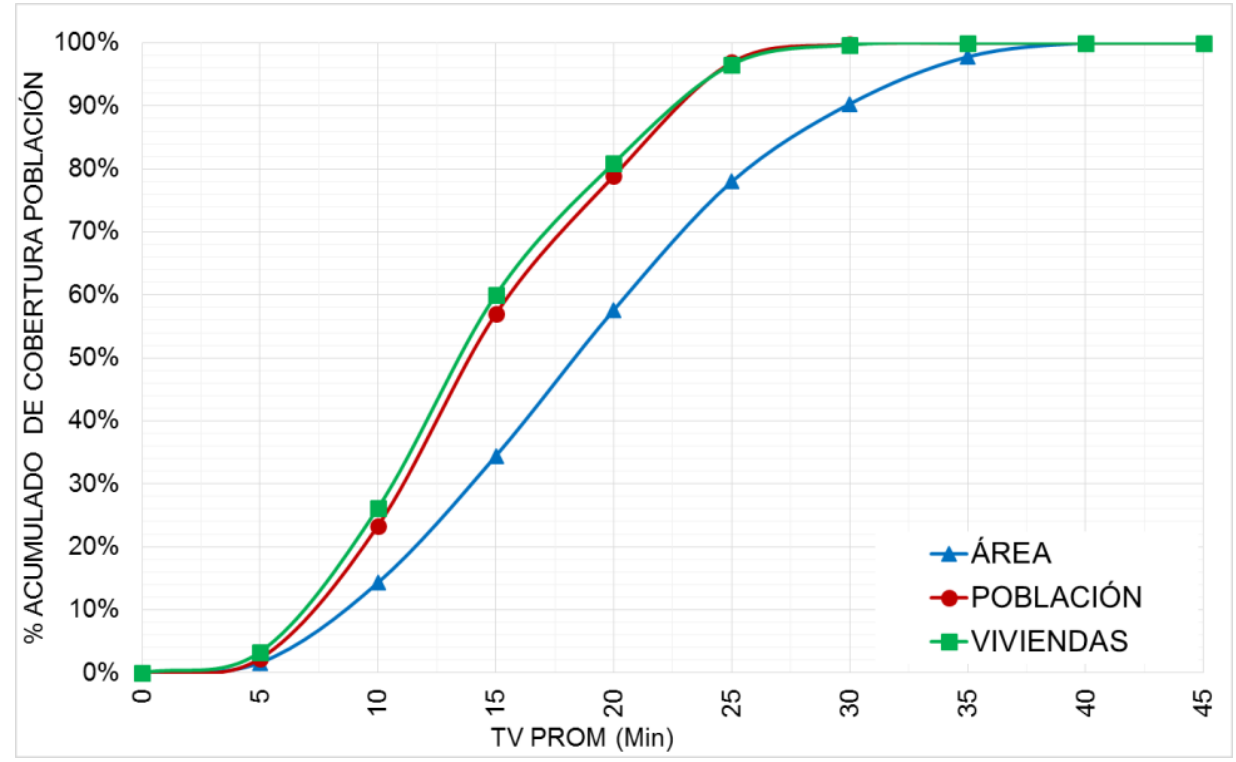

Fig. 6: Ojiva porcentual acumulada de las variables área, población y número de viviendas.

Tabla 2: Panorama Actual de la ciudad de Manizales.

\begin{tabular}{|l|l|l|l|l|l|}
\hline \multicolumn{1}{|c|}{ Escombrera } & \multicolumn{1}{|c|}{ Localización } & Área $\left(\mathrm{m}^{2}\right)$ & $\begin{array}{c}\text { Capacidad } \\
\left(\mathrm{m}^{3}\right)\end{array}$ & \multicolumn{1}{c|}{$\begin{array}{c}\text { Resolución Licencia } \\
\text { Ambiental No. }\end{array}$} & \multicolumn{1}{c|}{ Observación } \\
\hline 1. Villapilar & $\begin{array}{l}\text { Curva de la Nena - } \\
\text { Livonio }\end{array}$ & 40.780 & 211.939 & $1621(01 / 09 / 97)$ & Cumplió Vida Útil \\
\hline 2. El Bosque & Sur barrio el Bosque & 55.296 & 645.349 & $\begin{array}{l}\text { Términos de } \\
\text { Referencia }\end{array}$ & Cumplió Vida Útil \\
\hline 3. Villacarmenza & Barrio Villacarmenza & 66.365 & 728.634 & $1051(23 / 08 / 96)$ & $\begin{array}{l}\text { Cierre, falla en Box } \\
\text { Coulvert }\end{array}$ \\
\hline 4. Santos & $\begin{array}{l}\text { Entre barrios Guamal y } \\
\text { Eucaliptos }\end{array}$ & 27.999 & 279.712 & $1525(019 / 06 / 97)$ & Cumplió Vida Útil \\
\hline 5. Inem & $\begin{array}{l}\text { Al sur de los colegios } \\
\text { Inem y San Luis }\end{array}$ & 56.608 & 736.007 & $\begin{array}{l}\text { Términos de } \\
\text { Referencia }\end{array}$ & Cumplió Vida Útil \\
\hline 6. Bajo Palermo & $\begin{array}{l}\text { Entre barrios Betania, } \\
\text { Fátima y Bajo Palermo }\end{array}$ & 29.959 & 205.324 & $1540(23 / 06 / 97)$ & Cumplió Vida Útil \\
\hline
\end{tabular}

La anterior situación, llevó a las autoridades municipales a que en el año 2014 se vieran en la necesidad de habilitar otros sitios, ya que la ciudad de Manizales se enfrentaba a una fuerte crisis en relación con la poca oferta de lugares adecuados para la disposición de escombros (Gómez, 2014).

\section{Accesibilidad Media Integral a las Escombreras. Situación real (2016).}

La ciudad de Manizales ha sufrido en los últimos dos años grandes problemas para la disposición de residuos sobrantes de construcciones, en un artículo periodístico publicado en la prensa local (Gómez, 2014), se reflexiona sobre dicha problemática, llevando a que la administración municipal, en conjunto con el Ministerio de Medio Ambiente, expidieran el Decreto $N^{\circ} 0389$ de 2015, mediante el cual se adicionan escombreras nuevas al Plan de Ordenamiento Territorial vigente. Mediante el mencionado decreto se legalizó la adecuación y puesta a punto de una escombrera ubicada en el Barrio la enea (Finca la Granja), y otra en el Barrio La Playita, la cual operaba sólo para recibir otro tipo de residuos sólidos.

En la Fig. 7 se observan las curvas isócronas de Accesibilidad media integral obtenidas al aplicar la metodología de cálculo sólo con las tres escombreras que se encuentran realmente operando en el área de estudio, (La enea, la playita y Villamaría); se resalta que los tiempos medios de viaje necesarios para cubrir toda el área de estudio llegan hasta los 70 minutos, mientras que en el escenario de las escombreras legalmente establecidas por el POT, llegaban a los 40 minutos. Por otra parte, se observan los sitios que 
actualmente se relacionan con obras de construcción civil, encontrando que $60 \%$ se alcanzarían con tiempos de entre 5 y 45 minutos, mostrando la amplia diferencia entre lo que se encontraba legalizado por el POT y que realmente debe invertirse en tiempo para llegar a este tipo de actividad.

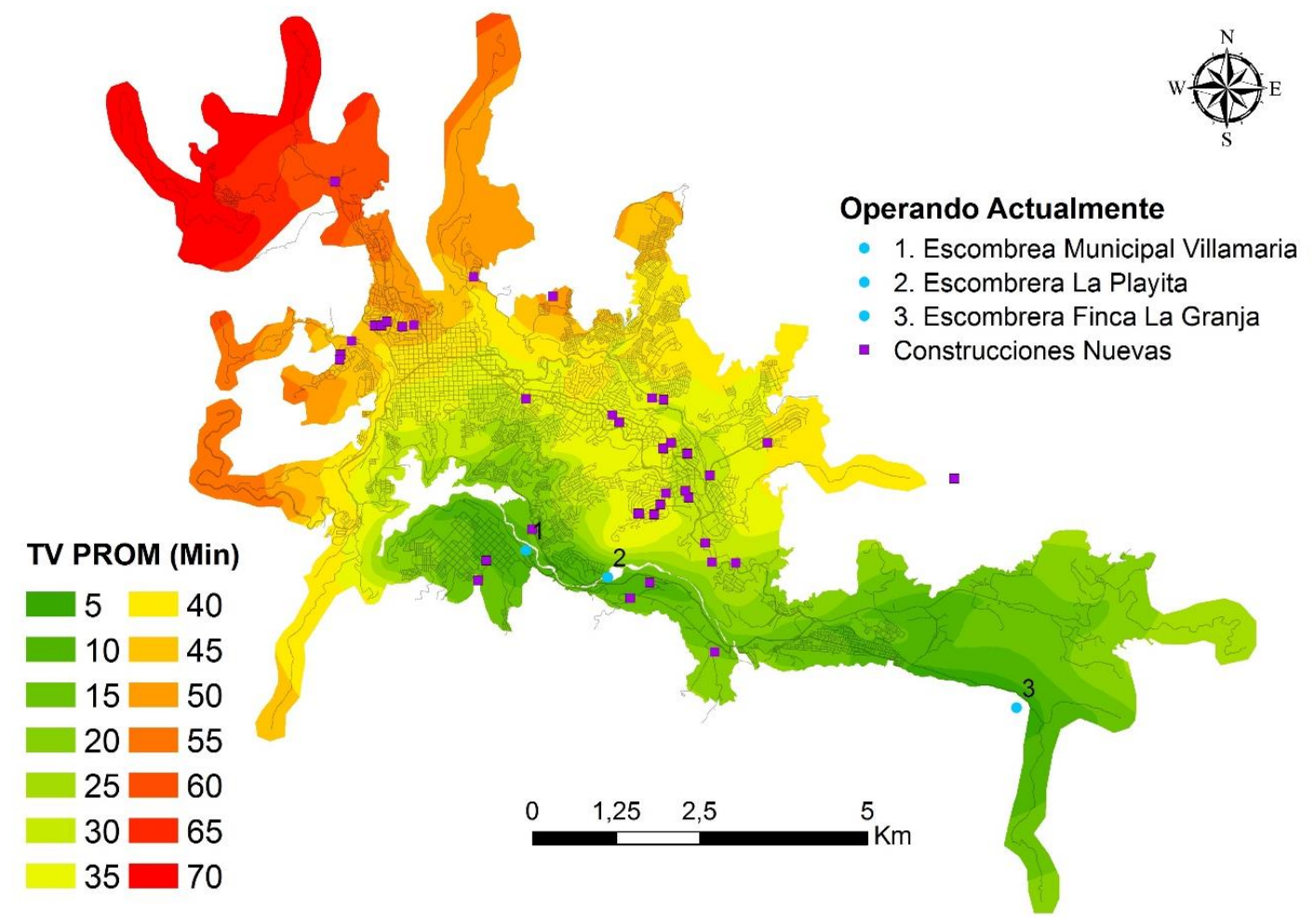

Fig. 7: Curvas de Accesibilidad Media Integral de la zona en estudio. Escenario actual.

Se encontró que las obras de construcción civil, en la actualidad, deben invertir mayor cantidad de tiempo del estimado para las escombreras vigentes en el POT vigente, lo que lleva a detectarse una importante deficiencia en la selección o adecuación de sitios aptos para esta actividad; finalmente, se estima que desde cualquier obra de construcción civil que en este momento se está ejecutando, se deben invertir como mínimo 45 minutos, aumentando en un $125 \%$ el tiempo que debe ser invertido si las escombreras designadas por el POT vigente estuviesen en funcionamiento.

En la Fig. 8 se presenta la distribución y variación de porcentajes de cobertura de las tres variables analizadas (área, población y número de viviendas), según las curvas isócronas de tiempo en intervalos de cinco minutos. Se observa que los mayores porcentajes de cobertura se obtienen para tiempos medios de viaje de entre 35 a 45 minutos para las tres variables.

Se aprecia en la Fig. 9 la ojiva porcentual acumulada de las variables área, población y vivienda que son cubiertas por las curvas isócronas. Es posible concluir que el $25 \%$ del área se cubre con tiempos medios de viaje de entre 15 y 20 minutos, mientras que el $25 \%$ de la población y vivienda se cubre con tiempos medios de viaje menores a los 25 minutos; así mismo, el 50\% del área se cubre a los 30 minutos y el 50\% de la población y la vivienda se cubren a los 30 minutos.

\section{CONCLUSIONES}

La ciudad de Manizales, en el panorama actual, se encuentra con una alta deficiencia de lugares aptos para el depósito de escombros; lo cual ha ocasionado desórdenes y malas planeaciones a la hora de ejecutar el transporte de material sobrante desde las obras nuevas hasta los sitios de depósito. Los tiempos mínimos de viaje que se toman en el panorama actual, son evidentemente mayores a los estimados con los sitios habilitados en el POT vigente para la ciudad de Manizales; por otra parte, las escombreras habilitadas actualmente, se han ejecutado bajo normativas adicionales dada la deficiencia de sitios aptos para el uso de escombros y así generar una solución parcial para la ciudad.

Finalmente, la ciudad de Manizales está requiriendo la adecuación de mayores sitios públicos para el depósito de residuos provenientes de construcciones nuevas; en síntesis, se propone el estudio de nuevas zonas aptas para el aprovechamiento del suelo y realizar los depósitos de escombros en lugares indicados 
para ello, que luego presten un servicio a la ciudad, como por ejemplo la creación de parques recreativos en zonas donde las escombreras hayan cumplido su vida útil.

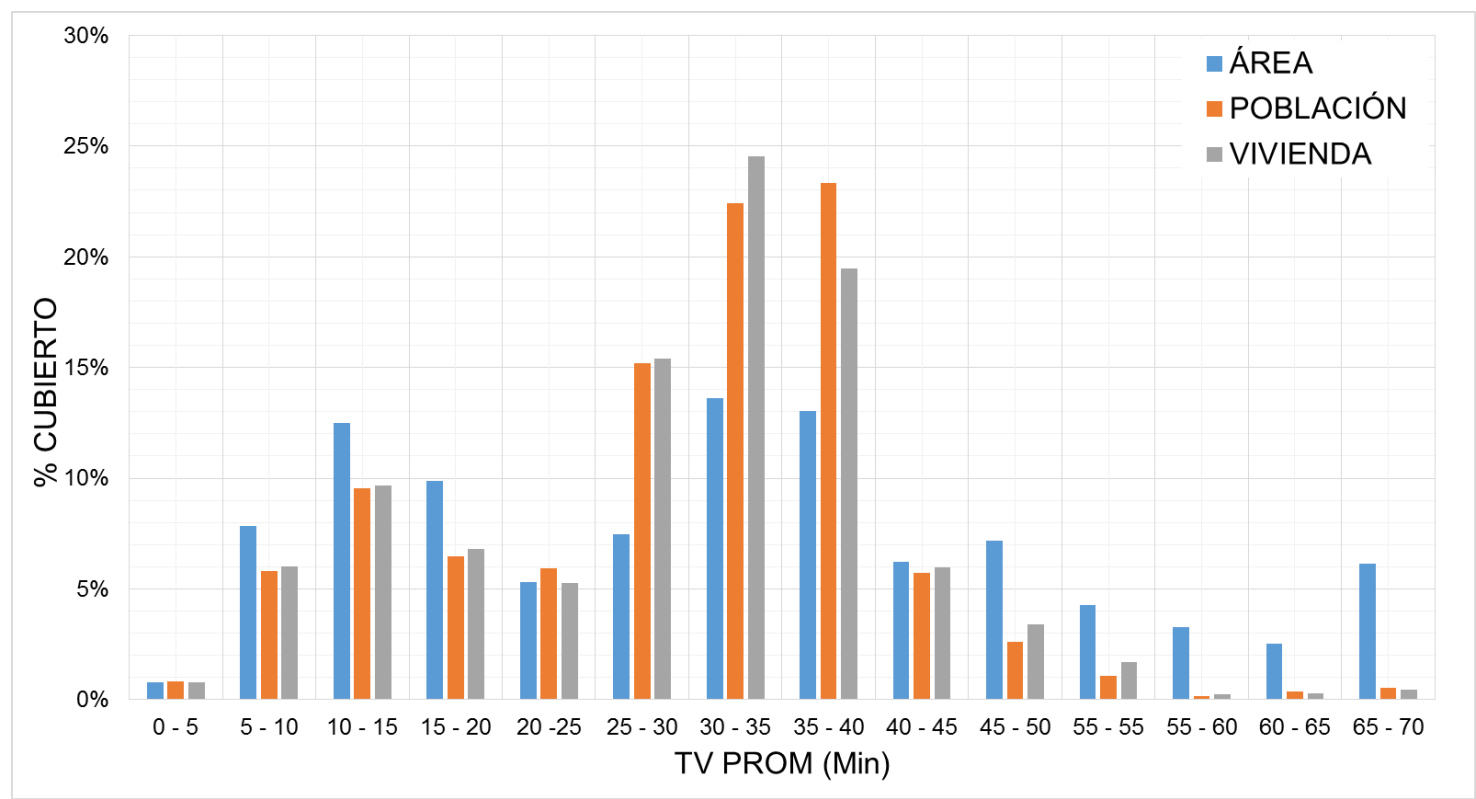

Fig. 8: Distribución porcentual de área, población y vivienda, cubiertas por las curvas isócronas.

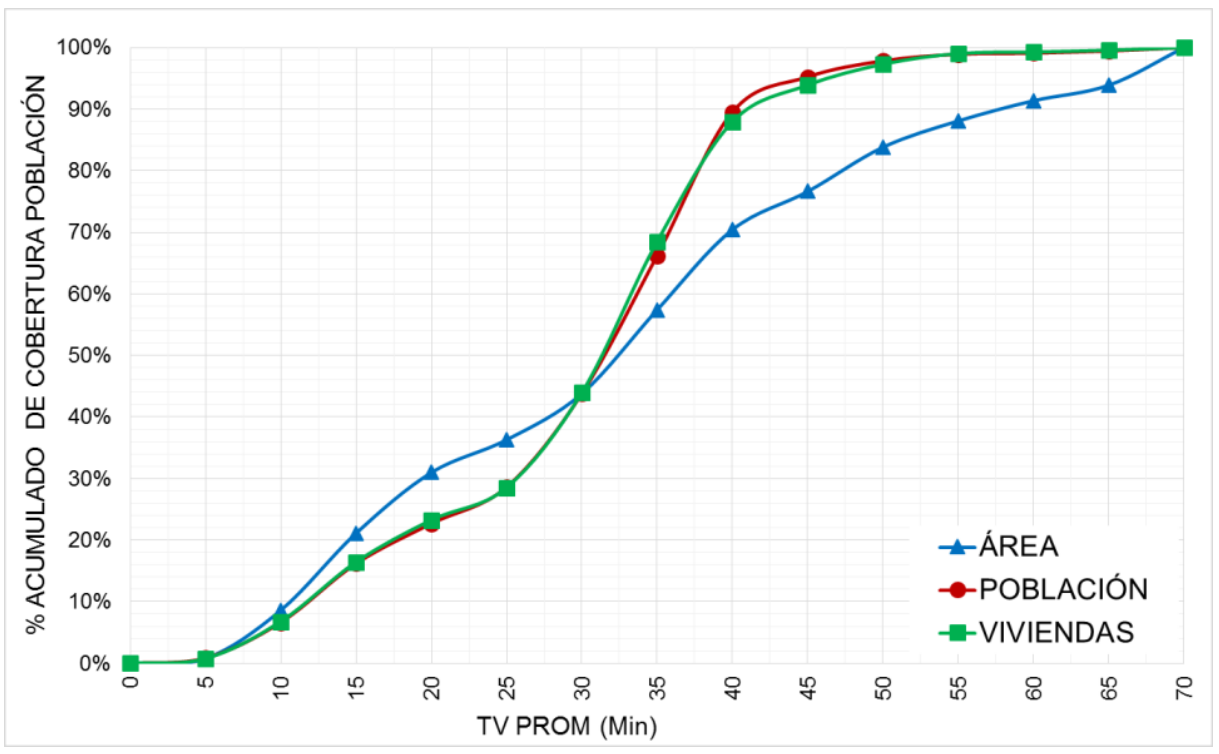

Fig. 9: Ojiva porcentual acumulada de las variables área, población y número de viviendas.

\section{AGRADECIMIENTOS}

Los autores expresan su agradecimiento a los estudiantes pertenecientes al Semillero de Investigación en Movilidad Sostenible y al Semillero de Investigación en Planificación Urbana, ambos de la Facultad de Ingeniería y Arquitectura de la Universidad Nacional de Colombia - Sede Manizales.

\section{REFERENCIAS}

Achillas, Ch., Moussiopoulos, N., Karagiannidis, A., Banias, G., y Perkoulidis, G.; The use of multi-criteria decision analysis to tackle waste management problems: a literature review, doi: 10.1177/0734242X12470203, Waste Management \& Research, 31(2), 115-129 (2013) 
Banias, G., Achillas, Ch., Vlachokostas, Ch., Moussiopoulos, N. y Tarsenis, S.; Assessing multiple criteria for the optimal location of a construction and demolition waste management facility, http://dx.doi.org/10.1016/j.buildenv.2010.04.016, Building and Environment, 45(10), 2317-2326 (2010)

Barros, A.I., Dekker, R. y Scholten, V.; A two-level network for recycling sand: A case study, doi:10.1016/S0377-2217(98)00093-9, European Journal of Operational Research, 110(2), 199-214 (1998)

Batty, M.; Accessibility: in search of a unified theory, doi: 10.1068/b3602ed, Environment and Planning B: Planning and Design, 36, 191-194 (2009)

Botero, R., Murillo, J. y Jaramillo, C.; Decision strategies for evaluating pedestrian accessibility, http://www.scielo.org.co/, ISSN: 0012-7353, Revista Dyna, 78 (168), 28-35 (2011)

Castaño, J., Rodríguez, R., Lasso, L., Cabrera, A. y Ocampo, M.; Gestión de residuos de construcción y demolición (RCD) en Bogotá: perspectivas y limitantes, Tecnura, 17, 121-129 (2013)

Coelho, A. y De Brito, J.; Economic viability analysis of a construction and demolition waste recycling plant in Portugal - part l: location, materials, technology and economic analysis, http://dx.doi.org/10.1016/j.jclepro.2012.08.024, Journal of Cleaner Production, 39, 338-352 (2013)

Curl, A., Nelson, J. y Anable, J.; Does Accessibility Planning address what matters?, doi.org/10.1016/j.rtbm.2012.04.003, Research in Transportation Business \&amp; Management, 3, 84 (2012)

Decreto - 0389, Reglamenta la adición de escombreras nuevas al plan vigente de ordenamiento territorial, Ministerio de medio ambiente (24 julio 2015), Manizales - Caldas (2015)

Empitalito E.S.P.; Estudio técnico, económico, financiero y jurídico para la implementación de una escombrera en el municipio de Pitalito, Huila, Empresa de Servicios Públicos Domiciliarios de Pitalito (2014)

Escobar, D., García, F. y Tolosa, R.; Diagnóstico de la Movilidad urbana de Manizales, 1a Edición, Universidad Nacional de Colombia, Facultad de Ingeniería y Arquitectura, Manizales, Colombia (2012)

Escobar D., García F. y Tolosa R.; Análisis de Accesibilidad Territorial a Nivel Regional, 1a Edición, Universidad Nacional de Colombia, Facultad de Ingeniería y Arquitectura, Manizales, Colombia (2013)

Escobar, D.A., Tapasco, O.A. y Giraldo, J.A.; Medición de Desempeño del Sistema de Transporte Cable Aéreo de la Ciudad de Manizales en Colombia, usando Tres Enfoques: Analítico, Simulado y de Accesibilidad Urbana, doi: 10.4067/S0718-07642015000600020, Información Tecnológica, 26(6), 199-210 (2015)

Geurs, K., Boon, W. y Van Wee, B.; Social impacts of transport: literature review and the state of the practice of transport appraisal in the Netherlands and the United Kingdom, doi: 10.1080/01441640802130490, Transport reviews, (en línea: https://goo.gl/ZlgSSK, acceso: 10 de noviembre de 2015), 29 (1), 69-90 (2009)

Gómez, M.; Manizales se quedó sin escombreras (en la web: https://goo.gl/4lhqad, acceso: 10 de mayo 2016), Periódico la Patria (2014)

Halden, D.; The use and abuse of accessibility measures in UK passenger transport planning, doi:10.1016/j.rtbm.2011.05.001, Transportation Business \& Management, 2, 12-19 (2011)

Hernández, J., Rivera, I., Patiño, F y Juárez, J.; Estudio Cinético de la Lixiviación de Plata en el Sistema S2O32--O2-Cu2+ Contenido en Residuos Minero-Metalúrgicos, doi: 10.4067/S0718-07642013000100007, Información Tecnológica, 24(1), 51-58 (2013)

Kastenholz, E., Eusébio, C., Figueiredo, E. y Lima, J.; Accessibility as Competitive Advantage of a Tourism Destination: The Case of Lousã, in Field Guide to Case Study Research in Tourism, Hospitality and Leisure (Advances in Culture, Tourism and Hospitality Research, Vol. 6, K.F. Hyde, C. Ryan and A.G. Woodside (Ed.), doi: 10.1108/S1871-3173(2012)0000006023, Emerald Group Publishing Limited, 369-385 (2012)

Khan, D. y Samadder, S.R.; Municipal solid waste management using Geographical Information System aided methods: A mini review, doi: 10.1177/0734242X14554644, Waste Management \& Research, 32(11), 1049-1062 (2014) 
Kotavaara, O., Antikainen, H. y Rusanen, J.; Population change and accessibility by road and rail networks: GIS and statistical approach to Finland 1970-2007, doi:10.1016/j.jtrangeo.2010.10.013, Journal of Transport Geography, 19 (4), 926-935 (2011)

Leandro, A.; Administración y manejo de los desechos en proyectos de construcción Etapa 2. Alternativas de Manejo., Informe de proyecto de investigación, Instituto Tecnológico, Costa Rica, Vicerrectoría de Investigación y Extensión (2007)

López, E., Gutierrez, J. y Gómez, G.; Measuring regional cohesion effects of large-scale transport infrastructure investment: an accessibility approach, doi: 10.1080/09654310701814629, European Planning Studies, 16 (2), 277-301 (2008)

Mackinnon, D., Pirie, G. y Gather, M.; Transport and economic development. In R. Knowles, J. Shaw, \& I. Docherty, Editors, Transport Geographies: Mobilities, Flows and Spaces (10-28), Blackwell Publishers, Oxford (2008)

Morris, J., Dumble, P. y Wigan, M.; Accessibility indicators in transport planning, doi:10.1016/01912607(79)90012-8, Transportation Research, A, 13, 91-109 (1978)

Phatarapon V., Pongtep P., Suparda K y Nuchananporn P.; A comparison of Spatial Interpolation Methods for predicting concentrations of Particle Pollution (PM10), International Journal of Chemical, Environmental \& Biological Sciences (IJCEBS) ISSN 2320-4087, 3 (4) (2015)

PGIRS., Plan de gestión integral de residuos sólidos, Metodología de la resolución 0754 del Ministerio de vivienda, cuidad y territorio, Ministro de ambiente y desarrollo sostenible, Villamaría - Caldas (2015)

POT., Plan de Ordenamiento Territorial Vigente, (en la web: https://goo.gl/II7XN6), Componente urbano, Manizales - Caldas (2007)

Sailer, K., Marmot A. y Penn, A.; Spatial Configuration, Organisational Change and Academic Networks, ASNA 2012 - Conference for 'Applied Social Network Analysis', Zürich, Suiza 4 a 7 Septiembre (2012)

Sánchez., E.; Rebajan tarifas en escombrera municipal en Neiva: (en la web: https://goo.gl/dFwHHu, acceso: 15 de noviembre 2016), Diario Extra (2013)

Serrano, M., Pérez, D.; Propuesta de un programa de gestión integral de escombros; II Simposio Iberoamericana de Ingeniería de Residuos, Universidad del Norte, Barranquilla (2009)

Shen, G.; Measuring Accessibility of Housing to Public-community Facilities Using Geographical Information Systems, doi: 10.1111/1467-940X.00056, Review of Urban \& Regional Development Studies 14(3), 235-255 (2002)

ThiKimOanh, L., Bloemhof-Ruwaard, J.M., CL Van Buuren, J., GAJ Van der Vorst, J. y Rulkens, W.H.; Modelling and evaluating municipal solid waste management strategies in a megacity: The case of Ho Chi Minh City, doi: 10.1177/0734242X15572177, Waste Management \& Research, 33(4), 370-380 (2015)

Tot, B., Srđević, B., Vujić, B., Tavares, M. y Vujić, G.; Evaluation of key driver categories influencing sustainable waste management development with the analytic hierarchy process (AHP): Serbia example, doi: 10.1177/0734242X16652963, Waste Management \& Research, 34(8), 740-747 (2016)

Yeheyis, M., Hewage, K., Alam, M.S., Eskicioglu, C. y Sadiq, R.; An overview of construction and demolition waste management in Canada: a lifecycle analysis approach to sustainability, doi: 10.1007/s10098-0120481-6, Clean Technologies and Environmental Policy, 15(1), 81-91 (2013)

Younes, C., Escobar, D.A. y Holguín, J.M.; Equidad, Accesibilidad y Transporte. Aplicación explicativa mediante un Análisis de Accesibilidad al Sector Universitario de Manizales (Colombia), doi: 10.4067/S071807642016000300010, Información Tecnológica, 27(3), 107-118 (2016)

Zhang, H. y Gao, Z.; Bilevel programming model and solution method for mixed transportation network design problem, doi: 10.1007/s11424-009-9177-3, Journal of Systems Science and Complexity, 22 (3), 446-459, (2009) 